\title{
The Phase Morphology of 2,5 DI-Substituted Pyridine Derivatives: A Quantum Mechanical Analysis
}

\section{Shailendar Kumar Thakur}

To cite this article: Shailendar Kumar Thakur (2014) The Phase Morphology of 2,5 DI-

Substituted Pyridine Derivatives: A Quantum Mechanical Analysis, Molecular Crystals and Liquid Crystals, 588:1, 28-40, DOI: 10.1080/15421406.2013.823336

To link to this article: http://dx.doi.org/10.1080/15421406.2013.823336

曲 Published online: 30 Dec 2013.

Submit your article to this journal ¿

Џll Article views: 39

Q View related articles $₫$

View Crossmark data $\nearrow$ 


\title{
The Phase Morphology of 2,5 DI-Substituted Pyridine Derivatives: A Quantum Mechanical Analysis
}

\author{
SHAILENDAR KUMAR THAKUR* \\ Department of Physics, Deen Dayal Upadhyay Gorakhpur University, \\ Gorakhpur, UP, India
}

\begin{abstract}
A comparative analysis of some mesogenic and nonmesogenic molecules of 2, $5 \mathrm{di}$ substituted pyridine derivatives molecules has been carried out. The multicenter multipole expansion method is used to obtain the various interaction energy terms. The phase morphology of the mesogenic molecular system has been further investigated with the aid of various energy terms of configurations. The multicenter multipole expansion method is satisfactory and predicts the mesogenic behavior of molecular systems.
\end{abstract}

Keywords Intermolecular interaction; mesophases; multicenter-multipole; phase transition temperature; Win-games 25 MAR $2010\left(\mathrm{R}_{2}\right)$

\section{Introduction}

Liquid crystals or mesogens have a wide range of applications in various fields and have attracted the attention of scientists [1]. Mesogens are very sensitive to the external parameters, and this sensitivity makes them useful in various applications such as display devices, photonic crystals, sensors, and biomedical applications [2-4]. Thermotropic liquid crystals can be categorized as nematic, cholesteric, and smectic [5]. The molecular phase behavior and appearance of phase sequences are decided by the entermolecular interaction energies $[1,2]$. Various properties and the molecular behavior of complex molecules or a system of organization of molecules at different physical condition can be interpreted with help of study of small mass liquid crystal molecule and their interaction energies [5].

There are many ways to control the physical properties of the molecular systems, for example, to introduce various polar and nonpolar groups in terminal positions and to vary the side chain in molecular systems [6]. Such a modification affects the interaction energies of interacting molecular systems. The aim of this study is to calculate the pair interaction energies of molecules for various configurations with interaction energy terms by variation in position and orientation of molecules, and to examine the range of energy components that appear in various phases. These energies are further used to analyze the phase morphology. The computation strategy to calculate interaction energy at various configurations of a molecular pair and methodology is documented in detail elsewhere [7-9, 10].

Four types of pyridine derivative molecules have been selected. These pyridine derivatives have variety of core and chain lengths. V. F. Petrove et al. [6] have produced a large

*Address correspondence to Shailendar Kumar Thakur, Department of Physics, Deen Dayal Upadhyay Gorakhpur University, Gorakhpur, UP, India. E-mail: sktup_in@ rediffmail.com 
Table 1. Important bond lengths, angle, and dihedral angle of Pd-1 molecule

\begin{tabular}{lccccc}
\hline \multicolumn{2}{c}{ Bond length $(\AA)$} & \multicolumn{2}{c}{ Angle $\left(^{\circ}\right)$} & \multicolumn{2}{c}{ Dihedral angle $\left(^{\circ}\right)$} \\
\hline C14-C17 & 1.498 & N13-C14-C17 & 116.38 & C1-C2-C3-C4 & 179.606 \\
C8 -C11 & 1.474 & C8-C11-C12 & 121.97 & C3-C4-C5-C6 & -68.0 \\
C4-C5 & 1.533 & C4-C5-C6 & 113.119 & C7-C8-C11-C17 & 31.324 \\
& & & & C12-N13-N14-C17 & 179.642 \\
\hline
\end{tabular}

number of 2, 5 di-substituted pyridine molecular systems with a variety of phase sequences, and four specific types have been selected, 4-butyl-(5-cyclohexane)-2-methyl pyridine (Pd-1), 4-hexane-1-4-di-nitro-4-cyclohexane-1-butoxy-4-phenyl(Pd-2), 4-hexane-1-nitro4-Cyclohexene-1-butoxy-phenyl (Pd-3), and 5-hexane 1-3-di-nitro-4-phenyl-1-butoxyphenyl (Pd-4) in brackets is the short name of the different compounds used throughout this paper. These compounds are very suitable to study about physics of mesophase formation. These days, the smectic phases are growing as the center of study as these molecules are finding more applications in new types of memory devices that are readable optically and display with video rates [11].

These calculations generated a large amount of data that are summarized for space economy.

\section{Result and Discussion}

\subsection{Geometry Optimization}

The geometry of all the molecules is optimized with the help of Win-games version, 25 MAR 2010 (R2) [12]. Geometry optimization, frequency analysis, and calculation of electrostatic properties were carried out using the hybrid density functional theory B3LYP with $6-31 \mathrm{G}^{* *}$ basis set without any constraint and is given below with numbering scheme.

(Abbreviation: $\mathrm{Cr}=$ crystal phase, $\mathrm{Sm}=$ smectic phase $\mathrm{N}=$ nematic phase, $\mathrm{I}=$ isotropic phase.)

The important bond lengths, angles, and the dihedral angles are given in the tables (Tables 1-4) for all the studied molecules. Obviously the molecules strongly deviate from the planar structure, except for Pd-4 and Pd-3 molecules.

Table 2. Important bond lengths, angle, and dihedral angles of the Pd-2 molecules

\begin{tabular}{lccccc}
\hline \multicolumn{2}{l}{ Bond length $(\AA)$} & \multicolumn{2}{c}{ Angle $\left({ }^{\circ}\right)$} & \multicolumn{2}{c}{ Dihedral Angle $\left(^{\circ}\right)$} \\
\hline C6-C7 & 1.409 & C1-C2-C3 & 113.053 & C1-C2-C3-C4 & 179.99 \\
C10-C13 & 1.350 & C6-C7-C8 & 114.227 & C5-C6-N7-C8 & -67.075 \\
C16-O19 & 1.329 & C9-N10-C13 & 120.126 & C9-C10-C13-C14 & -25.09 \\
O19-C20 & 1.374 & C15-C16-O19 & 116.134 & C15-C16-O19-C20 & -179.713 \\
C22-C23 & 1.481 & C16-O19-C20 & 118.438 & O19-C20-C21-C22 & -64.394 \\
& & O19-C20-C21 & 108.086 & & \\
\hline
\end{tabular}


Table 3. Important bond lengths, angle, and dihedral angles of the Pd-3 molecules

\begin{tabular}{lccccc}
\hline \multicolumn{2}{c}{ Bond length $(\AA)$} & \multicolumn{2}{c}{ Angle $\left({ }^{\circ}\right)$} & \multicolumn{2}{c}{ Dihedral Angle $\left.{ }^{\circ}\right)$} \\
\hline C6-C7 & 1.541 & C1-C2-C3 & & C1-C2-C3-C4 & 179.00 \\
C10-C13 & 1.402 & C6-C7-C8 & 114.002 & C5-C6-N7-C8 & -55.678 \\
C16-O19 & 1.370 & C9-N10-C13 & 118.93 & C9-C10-C13-C14 & 4.143 \\
O19-C20 & 1.422 & C15-C16-O19 & 116.371 & C15-C16-O19-C20 & -178.28 \\
& & C16-O19-C20 & 118.442 & O19-C20-C21-C22 & -63.664 \\
& & O19-C20-C21 & 108.063 & & \\
\hline
\end{tabular}

\subsection{Interaction Energy Calculation}

The interaction energy is given as $\mathrm{E}_{\mathrm{tot}}=\mathrm{E}_{\mathrm{el}}+\mathrm{E}_{\mathrm{pol}}+\mathrm{E}_{\mathrm{dis}}+\mathrm{E}_{\mathrm{rep}}$; all the energies are expressed in terms of $\mathrm{kcal} / \mathrm{mol}$ [10]; it includes electrostatic $\left(\mathrm{E}_{\mathrm{el}}\right)$, polarization $\left(\mathrm{E}_{\mathrm{pol}}\right)$, dispersion $\left(E_{\text {dis }}\right)$, and repulsion $\left(E_{\text {rep }}\right)$ energy terms. We have tabulated the energy components of all the studied molecules. The $\left(\mathrm{E}_{\mathrm{el}}\right)$ electrostatic energy terms includes monopole-monopole interaction term $\left(\mathrm{E}_{\mathrm{qq}}\right)$, monopole-dipole interaction term $\left(\mathrm{E}_{\mathrm{qni}}\right)$ and dipole-dipole interaction term $\mathrm{E}_{\operatorname{mini}}[10]$.

(a) $P d-1$ Molecule. The stacking configurations are parallel and antiparallel having large energy difference. Stacking-2 and plane side-2 are antiparallel having high energy while stacking-1 and plane side-1 are antiparallel with low energy (Fig. $2 \&$ Table 5). In electrostatic contributions, one of the stacking and one of the plane-side energy is positive also in two stacking and one of the plane-side energy contribution by dipole-dipole interaction is positive.

(b) Pd-2 Molecule. The Pd-2 molecule is larger than the Pd-1 molecule having two smectic phases. In this molecule, the cyclohexane ring has nitrogen substituted at the two connecting points of cyclohexane ring. The geometry of molecule is also deviated from the plane structure (Fig. 1 (b) \& Table 2).

The two stacking configurations have small energy difference that indicates the proper stacking. In plane-side interaction this difference is large. One of the plane-side energy is higher (negative) than the other stacking and plane-side configuration energies (Table 6). The monopole-monopole interaction energy in stacking-2 and plane side- 1 is positive. Monopole-dipole interaction energies except stacking-1 are very favorable and the dipole-dipole interaction energy except stacking side-2 is positive (Figure 3 ).

Table 4. Important bond lengths, angle, and dihedral angles of the Pd-4 molecules

\begin{tabular}{lccccc}
\hline \multicolumn{2}{l}{ Bond length $(\AA)$} & \multicolumn{2}{c}{ Angle $\left({ }^{\circ}\right)$} & \multicolumn{2}{c}{ Dihedral Angle $\left({ }^{\circ}\right)$} \\
\hline C6-C7 & 1.482 & C6-C7-C8 & 122.866 & C5-C6-C7-C8 & -87.580 \\
N9-N10 & 1.326 & C9-C10-C13 & 117.485 & N9-C10-C13-C14 & 0.174 \\
N11-N12 & 1.330 & N11-C10-C13 & 117.879 & C15-C16-O19-C20 & -179.62 \\
C10-C13 & 1.453 & C15-C16-O19 & 115.505 & O19-C20-C21-C22 & -64.0 \\
C16-C-19 & 1.338 & C19-O20-C21 & 107.720 & O20-C21-C22-C23 & 179.564 \\
C19-C20 & 1.406 & & & & \\
\hline
\end{tabular}




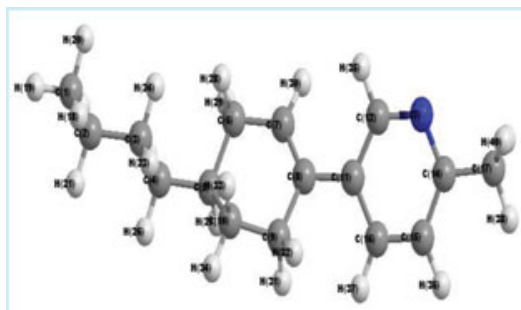

(a) $\mathrm{Pd}-1\left(\mathrm{Cr}-20^{\circ} \mathrm{C}-\mathrm{I}\right)$

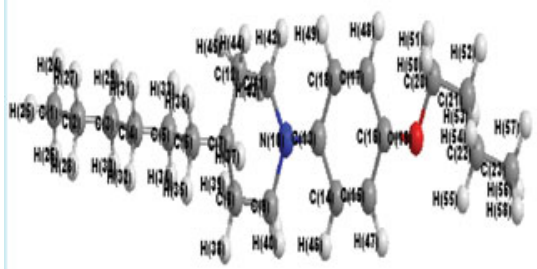

(c) Pd-3 (Cr-40.4-SmB-78-1)

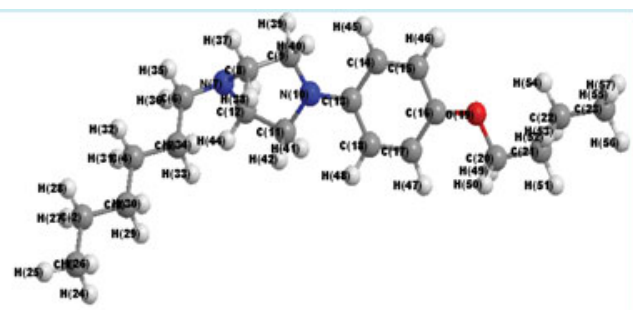

(b) Pd-2 (Cr-20.8-Sm-54.5-SmA-83.4-I)

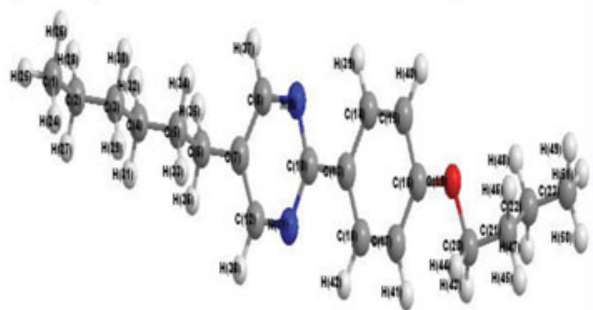

(d) Pd-4 (Cr-40.4-N-53-I)

Figure 1. Molecule structures with numbering scheme and phase sequences.

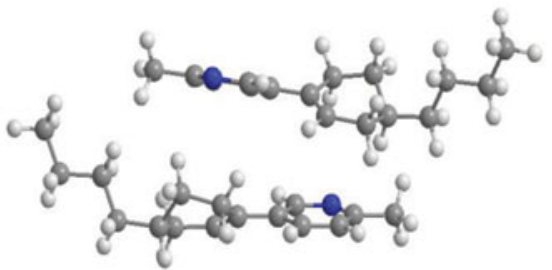

(a) Stacking-1 Configuration.

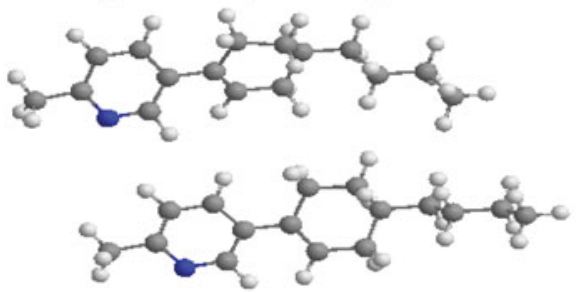

(c)Plane side-1 configuration.

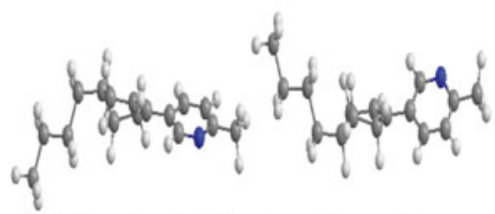

(e) Terminal side-1configuration.

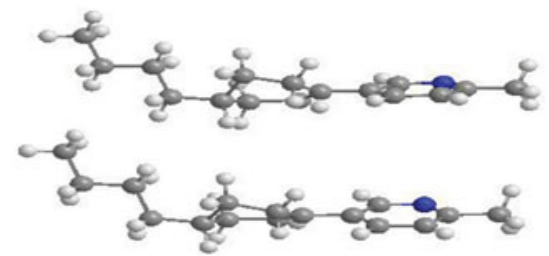

(b) Stacking-2 Configuration.

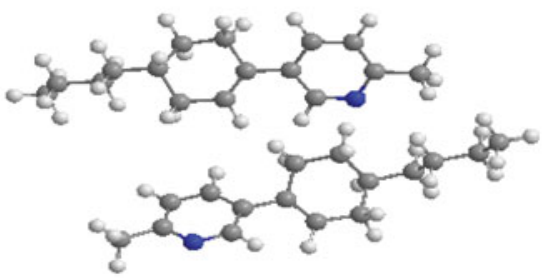

(d) Plane side-2 configuration.

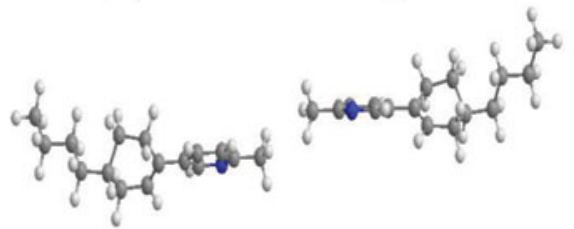

(f) Terminal side-2 configuration.

Figure 2. Various configurations for the Pd-1 molecule. 


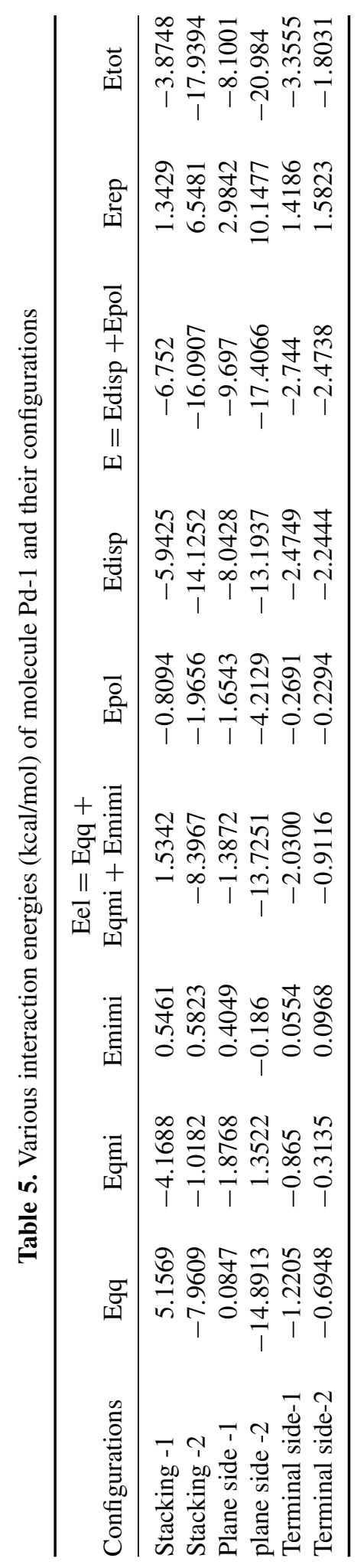




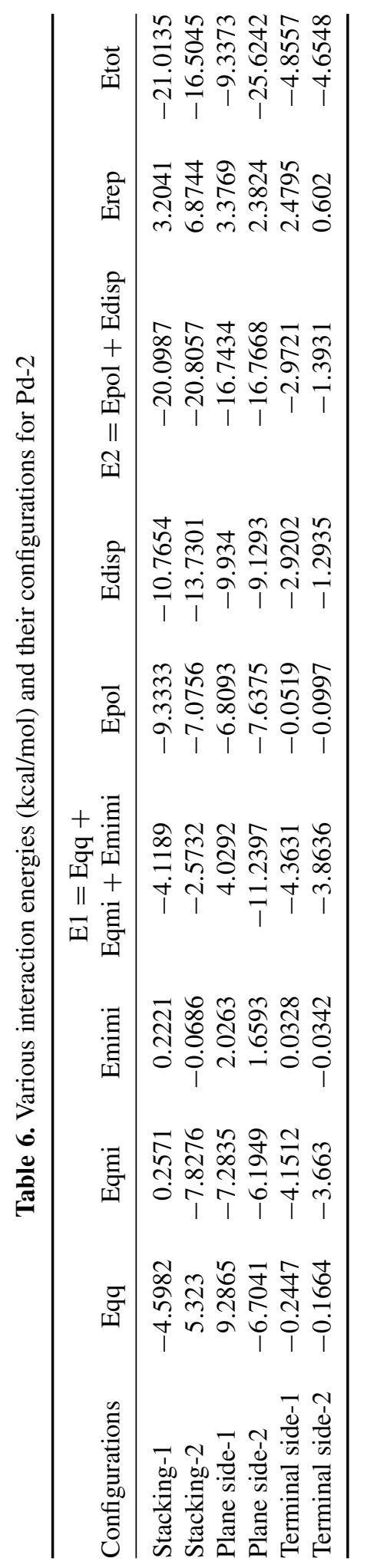




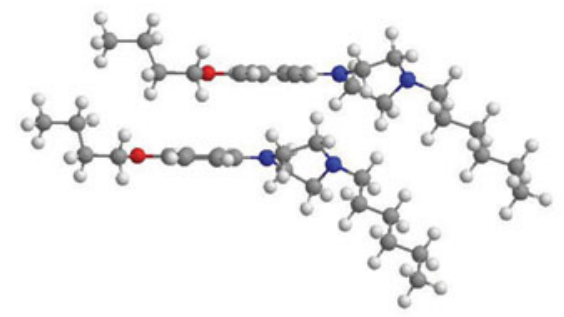

(a) Stacking-1 configuration.

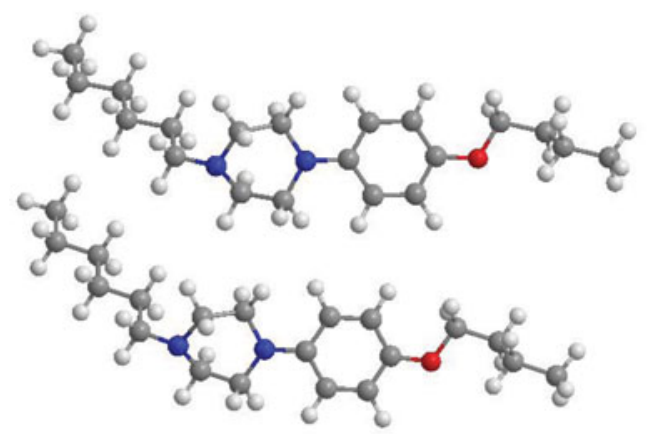

(c) Plane side-1 configuration.

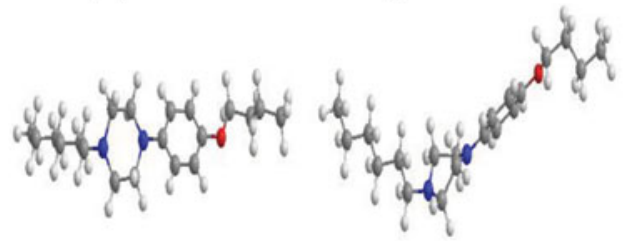

(e) Terminal side-1 configuration.

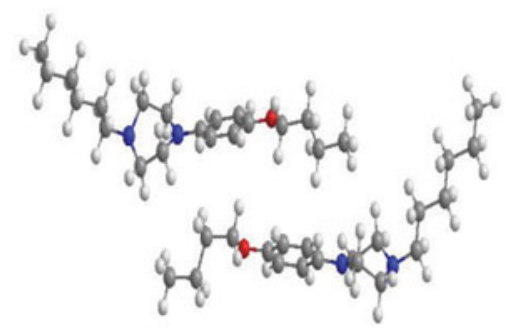

(b) Stacking-2 configuration.

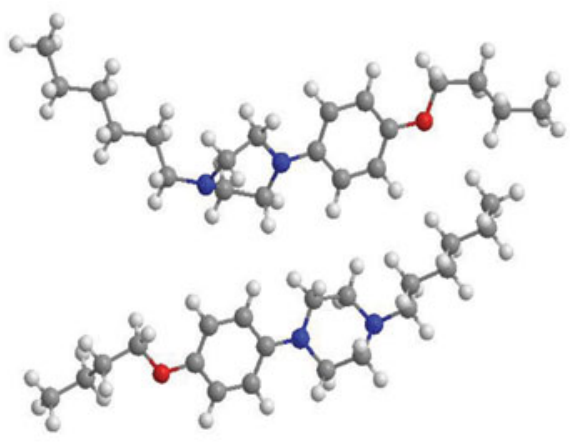

(d) Plane side-2 configuration.

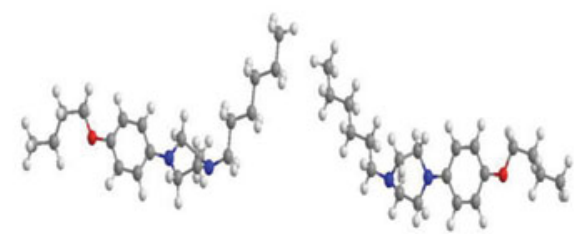

(f) Terminal side-2 configuration.

Figure 3. Interaction configurations for Pd-2 molecule.

(c) Pd-3 Molecule. This molecule has single nitrogen in cyclohexene ring at one of the connecting points. The nitrogen in Pd-3 is connected to phenyl-carbon while in Pd-2 molecule, one of the nitrogen, is providing local charge density. In Pd-3 molecule one of the stacking configuration energy is quite high. The high-stacking interaction with moderate plane-side interaction energy (Table 7 \& Fig. 4 (b)) causes the molecule to arrange in specific way. The monopole-dipole interaction energy for all the stacking and plane-side interaction is positive; also in stacking-2 configuration, the dipole-dipole interaction energy is positive while the monopole-monopole interaction energies are negative and large. These energies are long range and play effective role for the stability and formation of mesophase. This molecule comes to smectic phase at $40^{\circ} \mathrm{C}$ although it cannot be directly indicated from the interaction energies. The high-stacking energy indicates the formation of mesophase a bit large span of thermal range.

(d) Pd-4 Molecule. This molecule is nematic. From Table 8 it is obvious that the longrange interaction energy is effectively favorable. The formation of nematogen is possible only when the molecule should be in liquid state along with orientational arrangement 


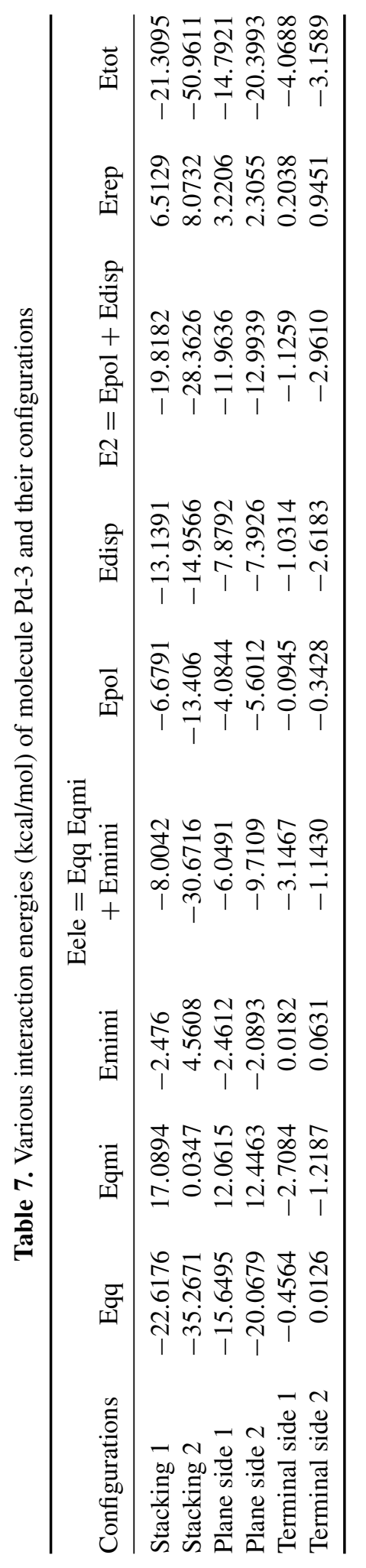




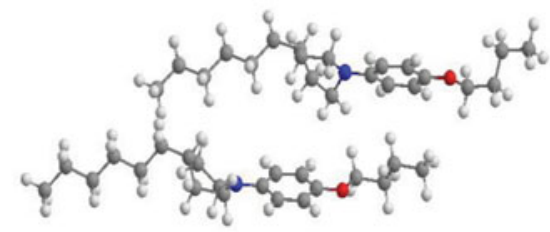

(a)Stacking-1 configuration.
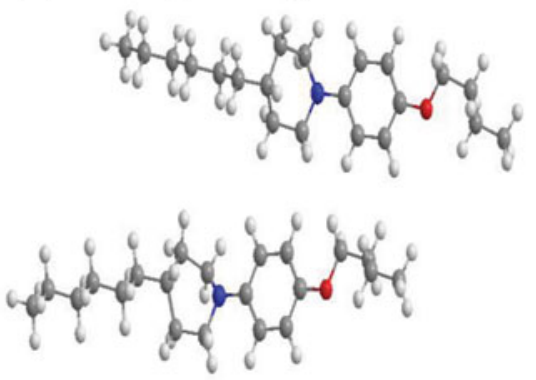

(c) Plane side-1 side configuration.

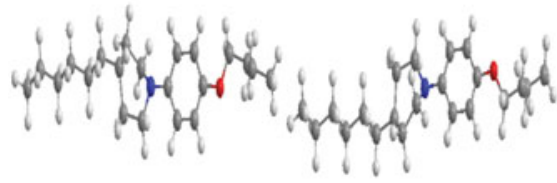

(e)Terminal side-1 configuration.

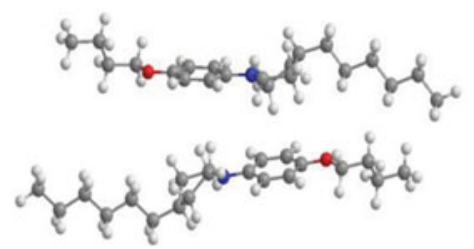

(b) Stacking-2 configuration.

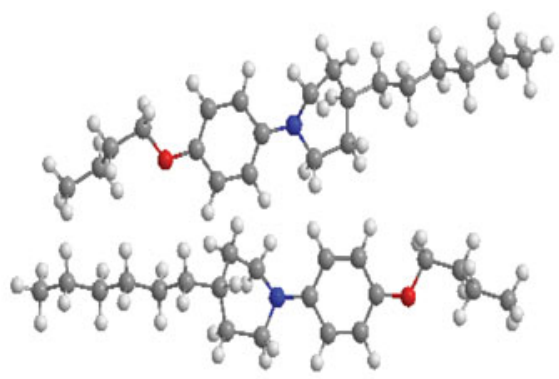

(d) Plane side-2 side configuration.

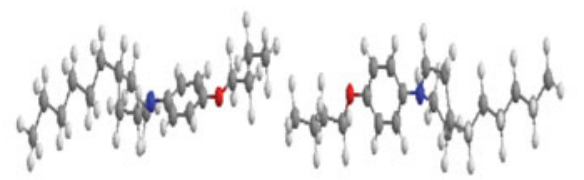

(f) Terminal side-2 configuration.

Figure 4. Various configurations for Pd-3 molecule.

in specimen. The electrostatic energies component monopole-dipole interactions are positive. For the nematic phase, long-range interactions are favorable (negative) while the monopole-dipole interactions are positive in magnitude of moderate value; also the similar pattern exists in dipole-dipole interaction in this molecule. From figure 8 it is obvious that in two stacking configurations, ring-ring, overlap each other. Also plane-side, $\mathrm{N}$ to $\mathrm{H}$ interaction, provide the molecular alignment along the particular direction in bulk specimen because these interactions are observed in both of the in-plane interactions.

In Pd-1 molecule, from Table 5 and Figure 2, it is obvious that stacking- 2 and plane side- 2 are preferred configurations in absence of thermal energy. These two orientations are parallel and antiparallel to each other. In bulk specimen, all the configurations are present. Those molecules, which are in specimen, have plane side- 1 and stacking- 1 configuration form weak interactions zone for parallel and antiparallel orientation. For a little rise in temperature, the parallel or antiparallel orientations are disturbed. It is also supported by Eqq. Thus by the interaction energy study, it is hard that molecule can form any mesophase.

In Pd-2 molecule, except one of the plane-side configuration, all the other plane and stacking configurations energetically plausible to form the smectic configuration. The Eqq for configurations stacking- 2 and planeside- 1 is positive with large magnitude. This may be the cause that the molecule has isotropic phase (Figure 3).

In Pd-3 molecule, the stacking- 2 configuration is energetically very favorable and in this interaction nitrogen from one of the molecule and ring of another molecule are interacting together. It is a preferred configuration present in crystal and smectic-B phase. 


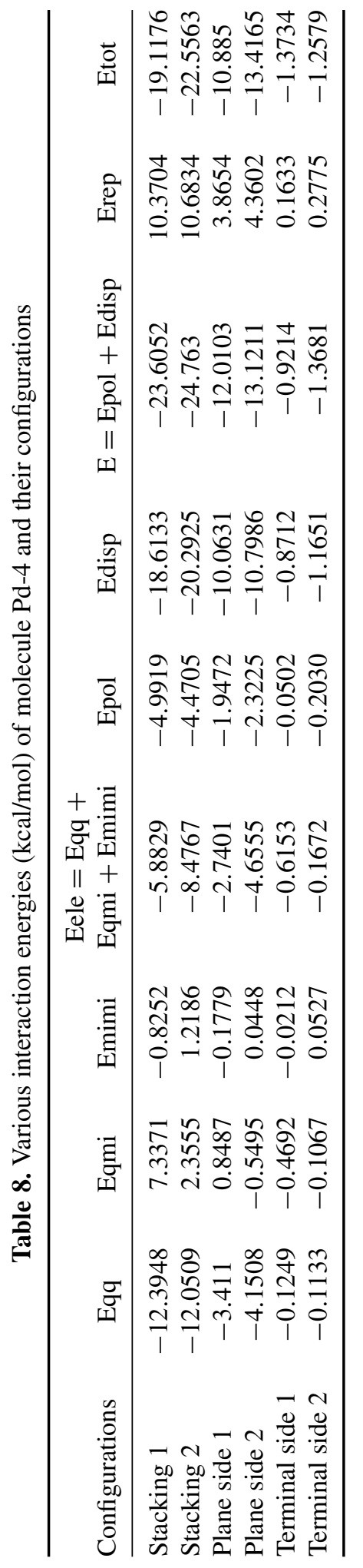




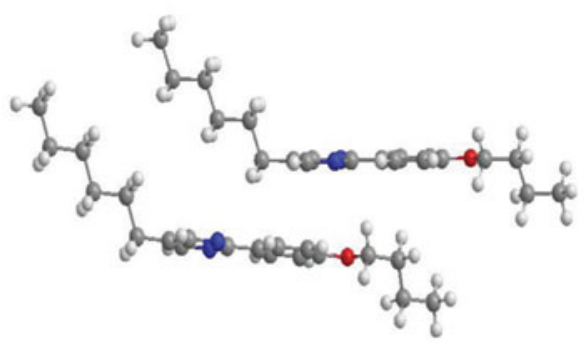

(a) Stacking-1 configuration.

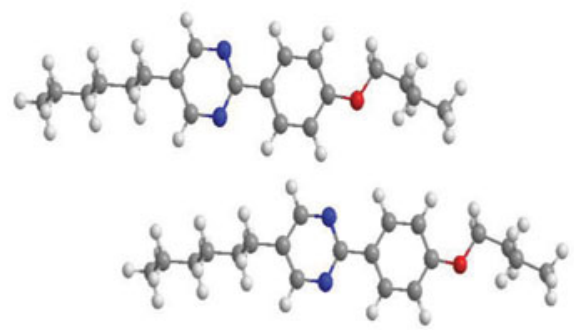

(c) Plane side-1 configuration.

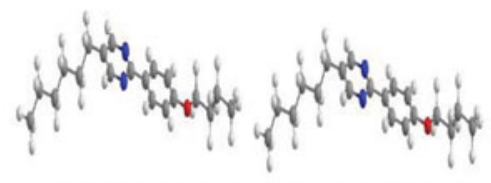

(e) Terminal side-1 configuration.

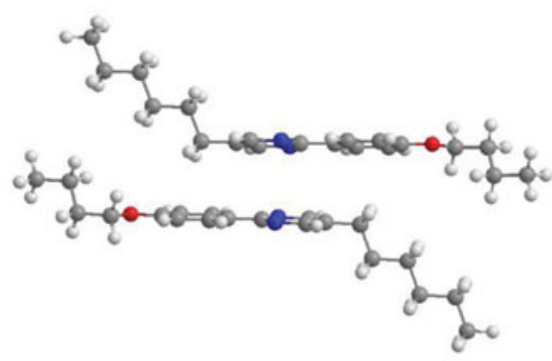

(b) Stacking-2 configuration.

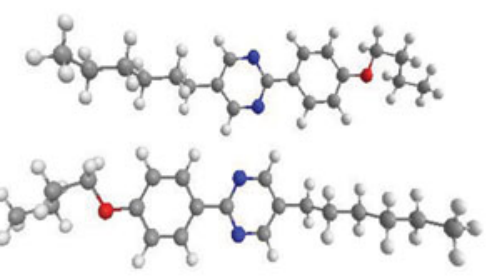

(d) Plane side-2 configuration.

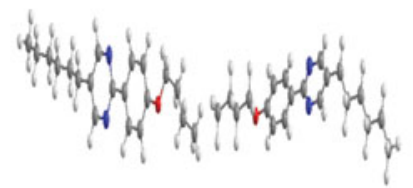

(f) Terminal side-2 configuration.

Figure 5. Interaction configurations for Pd-2 molecule.

$\mathrm{E}^{\mathrm{qq}}$ in plane side and stacking configurations is negative, and $\mathrm{E}_{\mathrm{qni}}$ is positive and magnitude is a bit large. Presence energetic cause of nematic phase in this molecule is very small.

The $\mathrm{E}_{\mathrm{qq}}$ interaction energies in electrostatic interaction are negative for all stacking and plane-side interaction configurations for Pd-4 molecule. While the monopole-dipole interaction energies in stacking and plane-side configurations are positive except one of the plane side interaction (Table 8). In stacking configurations the benzene ring or the pyridine ring of one molecule is properly overlapping with other molecules' benzene or pyridine ring (Figure 5).

Table 9. Comparison of various energy $(\mathrm{kcal} / \mathrm{mol})$ and molecule phase sequences

\begin{tabular}{lccc}
\hline $\begin{array}{l}\text { Compound } \\
\text { phase transition } \\
\text { temperature) }\end{array}$ & $\begin{array}{c}\text { Electrostatic } \\
\text { energy }\end{array}$ & $\begin{array}{c}\text { Short range } \\
\text { repulsion } \\
\text { energy }\end{array}$ & $\begin{array}{c}\text { Polarization } \\
\text { and dispersion } \\
\text { energy }\end{array}$ \\
\hline Pd-1 (Cr-20 ${ }^{\circ}$ C-I $)$ & -24.91 & 24.02 & -55.1641 \\
Pd-2 (Cr20.8-Sm54.5-SmA-83.4-I & -22.129 & 18.9193 & -98.179 \\
Pd-3 (Cr-40-SmB-78-I) & -58.7251 & 21.2611 & -77.1472 \\
Pd-4 (Cr-40-N-53-I) & -22.5377 & 29.7202 & -75.786 \\
\hline
\end{tabular}


In Table 9 I have summarized the electrostatic, short-range repulsion and short-range attraction energy for all the molecules. In nematic phase the value of short-range repulsion terms is higher than the electrostatic long-range attraction term. In isotropic and the smectic phase, the electrostatic attraction energy is higher than the repulsion energy while in all the cases dispersion energy plays dominant role. For small molecule, the dispersion interaction is weak and unlikely to form ordered phase at room temperature. At a distance of 3 to $4 \AA$ of inter molecular separation the dispersive attraction energy in between two large molecules is fairly larger than $k T$ ( $k$ is Boltzmann constant and $T$ is absolute temperature) at room temperature so the molecule can be in a condensed phase due to London dispersion force. In the entire studied molecule, the dispersion plays dominant role but itself this force cannot distinguish the formation of different phases.

The short-range repulsive forces originated from the Pauli exclusion force, when the electron cloud of two molecules approache each other too closely. In properly stacked molecule, where the electron-electron interactions are feasible, this interaction is high and creates fluctuation. The dispersion forces are also created by correlation of fluctuating dipole formed by motion of electron around nucleus, so these two forces create a fluctuating environment and cause the nematic flow. In studied nematic phase, molecular interaction energy is appearing in this way (Table 9) that it may not be case for all nematic phase but there should be a particular span of short-range repulsion and long-range attraction energy in which molecules have the behavior of nematic phase. Strong electrostatic attraction ceases the fluctuation. This is observed in the case of Pd-2 and Pd-3 molecules. In the case of $\mathrm{Pd}-1$, the attractive force is slightly grater than the repulsive force, so here the fluctuation is present due to dispersion and short-range repulsion interactions in less strength but overall the condense phase environment is dominant due to mild dispersion energy. The dispersion energy is comparatively small in magnitude with respect to all other molecules studied here. Due to less strength of dispersion energy (I have discussed the causes of nonformation of alignment of molecular system in this case above) molecular alignment is not favored. Thus the phase remains liquid and order is not maintained.

In the case of two interacting molecules (have various sites of active atoms) if there are many local strong interactions available then the molecular alignment is strong and interaction forms the stacked layered structure. In this case, the smectic phases are more likely. Although in all the smectic phases this type of interaction need not to be present. The strong attractive (dispersion) interaction is sufficient to form the case of smectic phase. In the studied case, the two molecules Pd-2 and Pd-3 are forming smectic phase; the attractive interaction (long range and short range) are dominant, and do not cause intermolecular fluctuation up to permissible level to form the nematic flow.

\section{Conclusion}

All the selected molecules are pyridine derivatives. In nonmesogenic molecule, the asymmetry of interaction energy is major deciding factor in the studied case. In nematic phase, the positive contribution of short-range forces is greater than the electrostatic attraction energy and the contribution of dispersion energy range plays very crucial role. In smectic and nematic phases the attractive and dispersion energies are important factors. Thus the electrostatic interaction along with the short range energy components is the deciding factor for mesophase formation. For any pair of molecular configuration interaction the $\mathrm{E}_{\mathrm{qq}}, \mathrm{E}_{\mathrm{qni}}$ or $\mathrm{E}_{\text {mini }}$ is positive and the stability of perfect stacking or plane-side interaction becomes weak. Further investigation on other molecular systems is required for better insight as the interactions decide the phase of a molecule and phase morphology within a molecular system. 


\section{References}

[1] Georgiy, V. Tkachenko, (Ed.). (2009). New Developments in Liquid Crystals, I-Tech: Vienna, Austria.

[2] Maurice, Kleman, \& Oleg, D. Laverntovich. (2003). Soft Matter Physics: An Introduction, Springer-Verlag: New York, Ch. 2, pp. 61-66.

[3] Rolando Ferrini. (2009). Liquid crystals into planar photonic crystals. In: Georgiy V. Tkachenko (Ed.), New Developments in Liquid Crystals, pp. 23-25, I-Tech: Vienna, Austria.

[4] Gerardo, A. López Muñoz, \& Gerardo, A. Valentino Orozco. (2009). Three dimensional temperature distribution analysis of ultrasound therapy equipments using thermochromic liquid crystal films. In: Georgiy V. Tkachenko (Ed.), New Developments in Liquid Crystals, p. 93, I-Tech: Vienna, Austria.

[5] de Gannes, P. G., \& Prost, J. (1993). Physics of Liquid Crystals, 2nd ed., pp. 5-20, 512-517, Oxford University Press: Oxford.

[6] Roychoudhury, M., \& Thakur, S. K. (2011). Mol. Cryst. Liq. Cryst., 548, 192-208.

[7] Roychoudhury, M., Thakur, S. K., \& Pankaj, Gaurav. (2011). J. Molecular Liquids, 161, 55-62.

[8] Thakur, S. K., \& Roychoudhury, M. (In Press). Mol. Cryst. Liq. Cryst., P.ID."LM144”.

[9] Petrove, V. F., \& Pavluchenko, A. I. (2002). Mol. Cryst. Liq. Cryst., 383, 63-79.

[10] Win-Games Version, 25 Mar 2010 (R2), IOWA State University; Schmidt, M. W., Baldridge, K. K., Boatz, J. A., Elbert, S. T., Gordon, M. S., Jensen, J. H., Koseki, S., Matsunaga, N., Nguyen, K. A., Su, S. J., Windus, T. L., together with Dupuis, M., \& Montgomery, J. A. (1993). J. Comput. Chem, 14, 1347-1363.

[11] Claverie, P. (1978). Elaborations of approximate formulas for interactions between large molecules: Applications in organic chemistry. In: Pullman, B. (Ed.), Inter Molecular Interactions From Diatomic to Biopolymers, J. Wiley \& Sons Ltd.: New York, pp. 217-226.

[12] Iam-Choon Khoo. (2007). Liquid Crystals, 2nd ed., John Wiley \& Sons, Inc.: New York, p. 221. 